\title{
POLARIZED PAIRS, LOG MINIMAL MODELS, AND ZARISKI DECOMPOSITIONS
}

\author{
CAUCHER BIRKAR AND ZHENGYU HU
}

\begin{abstract}
We continue our study of the relation between log minimal models and various types of Zariski decompositions. Let $(X, B)$ be a projective log canonical pair. We will show that $(X, B)$ has a log minimal model if either $K_{X}+$ $B$ birationally has a Nakayama-Zariski decomposition with nef positive part, or if $K_{X}+B$ is big and birationally has a Fujita-Zariski or Cutkosky-KawamataMoriwaki-Zariski decomposition. Along the way we introduce polarized pairs $(X, B+P)$, where $(X, B)$ is a usual projective pair and where $P$ is nef, and we study the birational geometry of such pairs.
\end{abstract}

\section{$\S 1$. Introduction}

We will work over an algebraically closed field $k$ of characteristic zero. In this paper, we continue our study of the relation between log minimal models and various types of Zariski decompositions. Let $(X, B)$ be a projective log canonical (LC) pair. The main result of [3] states that if $K_{X}+B$ birationally has a weak Zariski decomposition, then the pair has a log minimal model, assuming that the log minimal model program (LMMP) holds in lower dimension; in particular, this assumes termination of log flips in lower dimension. In this paper, we show that if we take a stronger form of Zariski decomposition, then we can construct a log minimal model without any extra assumptions in lower dimension. (For simplicity we state our results in the absolute projective case but everything can be formulated and proved in a similar way in the relative setting.) More precisely, we state the following.

TheOrem 1.1. Let $(X, B)$ be a projective LC pair with $K_{X}+B$ pseudoeffective. If $K_{X}+B$ birationally has a Nakayama-Zariski decomposition with nef positive part, then $(X, B)$ has a log minimal model.

Received April 12, 2013. Revised May 3, 2013. Accepted August 19, 2013.

First published online July 16, 2014.

2010 Mathematics Subject Classification. Primary 14E30.

(C) 2014 by The Editorial Board of the Nagoya Mathematical Journal 
The assumption of the theorem is that on some resolution $f: W \rightarrow X$ we have the Nakayama-Zariski decomposition $f^{*}\left(K_{X}+B\right)=P_{\sigma}+N_{\sigma}$ with $P_{\sigma}$ nef. (In general, $P_{\sigma}$ is only pseudoeffective.) For more details on the terminology and the proof, see Section 4.

Our next result concerns the Fujita-Zariski and Cutkosky-KawamataMoriwaki (CKM)-Zariski decompositions.

TheOREM 1.2. Let $(X, B)$ be a projective $L C$ pair such that $K_{X}+B$ is big. Then, the following are equivalent:

(1) $K_{X}+B$ birationally has a Fujita-Zariski decomposition;

(2) $K_{X}+B$ birationally has a CKM-Zariski decomposition;

(3) $(X, B)$ has a log minimal model.

The theorem is similar to the main result of Kawamata in [11] for Kawamata log terminal (KLT) pairs. For the proof, see Section 5.

In order to prove the above theorems, we consider a class of pairs beyond the traditional LC pairs. We look at pairs $(X, B+P)$ in which $(X, B)$ is a usual projective pair and where $P$ is a nef $\mathbb{R}$-divisor; we call the pair a polarized pair. Besides this article, polarized pairs appear in other contexts, for example, the base-point-free theorem, canonical bundle formulas in [13] and [9], proof of [5, Theorem 1.2], [6], and so forth. It is interesting to see if one can extend the birational geometry of usual pairs to the setting of polarized pairs. For example, the cone and contraction theorems and the existence of log flips hold for polarized pairs. One can ask whether one can run an LMMP on $K_{X}+B+P$ which terminates. One can ask many other questions, some of which are treated in Section 3.

Conjecture 1.3. Let $(X, B+P)$ be a $\mathbb{Q}$-factorial divisorially log terminal (DLT) polarized pair. Then, we can run an LMMP on $K_{X}+B+P$ which terminates.

By saying that $(X, B+P)$ is $\mathbb{Q}$-factorial DLT, we mean that $(X, B)$ is $\mathbb{Q}$-factorial DLT. Our first result concerning the conjecture is the following.

TheOREM 1.4. Let $(X, B+P)$ be a $\mathbb{Q}$-factorial DLT polarized pair. Assume either that $P$ or $N=K_{X}+B-P$ is a $\mathbb{Q}$-divisor, or that $N \geq 0$ with $\operatorname{Supp} N \subseteq \operatorname{Supp} B$. Moreover, assume that every LMMP on $K_{X}+B$ terminates. Then, for any $\alpha \geq 0$ we can run an LMMP on $K_{X}+B+\alpha P$ which terminates. Moreover, if $\alpha \gg 0$, then the LMMP is P-trivial. 
Here $P$-trivial means that $P$ is numerically trivial on every extremal ray in the process; in particular, this means that the nefness of $P$ is preserved in this case.

Corollary 1.5. Let $(X, B+P)$ be a $\mathbb{Q}$-factorial DLT polarized pair of dimension at most 3. Assume either that $P$ or $N=K_{X}+B-P$ is a $\mathbb{Q}$ divisor, or that $N \geq 0$ with $\operatorname{Supp} N \subseteq \operatorname{Supp} B$. Then, for any $\alpha \geq 0$ we can run an $L M M P$ on $K_{X}+B+\alpha P$ which terminates. Moreover, if $\alpha \gg 0$, then the LMMP is P-trivial.

Most probably the $\mathbb{Q}$-divisor condition in these results is unnecessary. In Section 3 we also touch upon limiting pairs, which are more general than polarized pairs. The following theorem shows that [6, Conjecture 1] hold.

TheOrem 1.6. Let $(X, B+P)$ be an $L C$ polarized pair where $B, P$ are $\mathbb{Q}$-divisors, and assume that $K_{X}+B+P$ is big. Further, assume that the augmented base locus $\mathbf{B}_{+}\left(K_{X}+B+P\right)$ does not contain any $L C$ center of $(X, B)$. If $K_{X}+B+P$ birationally has a CKM-Zariski decomposition, then the section ring $R\left(K_{X}+B+P\right)$ is a finitely generated $k$-algebra.

The proof is given in Section 5. If $(X, B+P)$ is $\mathbb{Q}$-factorial DLT, the proof is trivial, so the difficulty has to do with LC singularities.

In Section 6 of this paper, we outline a strategy to show that if every pseudoeffective LC pair of dimension less than or equal to $d$ has a weak Zariski decomposition, then every such pair has a log minimal model. This is an attempt to remove the termination assumption in the main theorem of [3].

\section{§2. Preliminaries}

Let $k$ be an algebraically closed field of characteristic zero fixed throughout this article. All the varieties will be over $k$ unless stated otherwise. For an $\mathbb{R}$-divisor $M=\sum m_{i} M_{i}$ on some variety, we define $\|M\|=\max \left\{m_{i}\right\}$.

\section{Pairs}

A pair $(X, B)$ consists of a normal quasiprojective variety $X$ and an $\mathbb{R}$-divisor $B$ on $X$ with coefficients in $[0,1]$ such that $K_{X}+B$ is $\mathbb{R}$-Cartier. For a prime divisor $D$ on some birational model of $X$ with a nonempty center on $X, a(D, X, B)$ denotes the $\log$ discrepancy. For definitions and standard results on singularities of pairs, see [14]. 
Log minimal models and Mori fiber spaces

A projective pair $\left(Y, B_{Y}\right)$ is a $\log$ birational model of a projective pair $(X, B)$ if we are given a birational map $\phi: X \rightarrow Y$ and $B_{Y}=B^{\sim}+E$, where $B^{\sim}$ is the birational transform of $B$ and $E$ is the reduced exceptional divisor of $\phi^{-1}$; that is, $E=\sum E_{j}$, where $E_{j}$ are the exceptional $/ X$ prime divisors on $Y$. A log birational model $\left(Y, B_{Y}\right)$ is a weak $L C$ model of $(X, B)$ if

- $K_{Y}+B_{Y}$ is nef, and

- for any prime divisor $D$ on $X$ which is exceptional $Y$, we have

$$
a(D, X, B) \leq a\left(D, Y, B_{Y}\right)
$$

A weak LC model $\left(Y, B_{Y}\right)$ is a log minimal model of $(X, B)$ if

- $\left(Y, B_{Y}\right)$ is $\mathbb{Q}$-factorial DLT, and

- the above inequality on log discrepancies is strict.

On the other hand, a log birational model $\left(Y, B_{Y}\right)$ is called a Mori fiber space of $(X, B)$ if

- $\left(Y, B_{Y}\right)$ is $\mathbb{Q}$-factorial DLT,

- there is a $\left(K_{Y}+B_{Y}\right)$-negative extremal contraction $Y \rightarrow T$ with $\operatorname{dim} Y>$ $\operatorname{dim} T$, and

- for any prime divisor $D$ (on birational models of $X$ ), we have

$$
a(D, X, B) \leq a\left(D, Y, B_{Y}\right)
$$

and strict inequality holds if $D$ is on $X$ and contracted/ $Y$.

Note that our definitions of log minimal models and Mori fiber spaces are slightly different from the traditional definitions in that we allow $\phi^{-1}$ to contract certain divisors.

\section{Rational decomposition of certain divisors}

Let $(X, B)$ be a $\mathbb{Q}$-factorial LC pair such that $K_{X}+B=P+N$, where $P$ is an $\mathbb{R}$-divisor and $N$ is a $\mathbb{Q}$-divisor. For each $\delta>0$, we will show that there is a decomposition $P=\sum r_{i} P_{i}$ satisfying the following:

- $P_{i}$ are $\mathbb{Q}$-Cartier,

- $r_{i}$ are positive and linearly independent over $\mathbb{Q}$,

- $\left\|P-P_{i}\right\|<\delta$ and $\operatorname{Supp}\left(P-P_{i}\right) \subseteq \operatorname{Supp} B$, and

- $\left(X, B_{i}\right)$ is LC where $B_{i}:=P_{i}+N-K_{X}$.

Let $V$ be the $\mathbb{R}$-vector space generated by the components of $K_{X}, B, P, N$. In what follows, a rational vector space (inside $V$ ) means an $\mathbb{R}$-vector space 
generated by a collection of $\mathbb{Q}$-divisors, and a rational affine space (inside $V$ ) means an affine space spanned by a collection of $\mathbb{Q}$-divisors.

Let $W \subseteq V$ be the smallest rational affine space containing $P$, and let $U$ be the vector space generated by the elements of $W$. If $Q \in W$, then $P-Q$ is supported on the irrational part of $P$, and since $N$ is a $\mathbb{Q}$-divisor, the irrational part of $P$ is supported in the irrational part of $B$; hence, $\operatorname{Supp}(P-Q) \subseteq \operatorname{Supp} B$.

First, assume that $W \neq U$. Then, we can write $P=\sum_{1}^{n} r_{i} P_{i}$, where $P_{i} \in W$ are $\mathbb{Q}$-divisors, $r_{i}>0$, and $n=\operatorname{dim} W+1=\operatorname{dim} U$. The $r_{i}$ are linearly independent over $\mathbb{Q}$; otherwise, we have $\sum a_{i} r_{i}=0$ for certain $a_{i} \in \mathbb{Q}$ (say, $a_{n}=1$ ). Hence, $P=\sum_{1}^{n-1} r_{i} P_{i}^{\prime}$, where $P_{i}^{\prime}=P_{i}-a_{i} P_{n}$, and this implies that $P$ belongs to a rational vector space strictly smaller than $U$, a contradiction. Now assume that $W=U$. In this case, we can choose $\mathbb{Q}$-divisors $P_{i}$ which form a basis of $W$ and such that $P$ belongs to the convex cone generated by the $P_{i}$; that is, $P=\sum_{1}^{n} r_{i} P_{i}$ for certain $r_{i}>0$. The $r_{i}$ are linearly independent over $\mathbb{Q}$; otherwise, $P$ would belong to a rational vector space strictly smaller than $W$, a contradiction. In both cases $W \neq U$ and $W=U$, it is obvious that we can choose the $P_{i}$ so that $\left\|P-P_{i}\right\|<\delta$.

It remains to show that the $P_{i}$ can be chosen so that $\left(X, B_{i}\right)$ is $\mathrm{LC}$, where $B_{i}:=P_{i}+N-K_{X}$. Put $W^{\prime}=W+N-K_{X}$, which is a rational affine space containing $B$. By Shokurov [19], the set

$$
\mathcal{L}=\left\{\Delta \in W^{\prime} \mid(X, \Delta) \text { is } \mathrm{LC}\right\}
$$

is a rational polytope. By our choice of $W, B$ belongs to the interior of $\mathcal{L}$; otherwise, $B$ would belong to some proper face of $\mathcal{L}$. Hence, there would be a rational affine space $T^{\prime} \subsetneq W^{\prime}$ containing $B$, which in turn implies that $P$ belongs to the rational affine space $T:=T^{\prime}+K_{X}-N \subsetneq W$, a contradiction. The same reasoning shows that $\operatorname{dim} \mathcal{L}=\operatorname{dim} W$. Therefore, if we choose $P_{i}$ so that $\left\|P-P_{i}\right\|=\left\|B-B_{i}\right\|$ is sufficiently small, then each $B_{i}$ belongs to the interior of $\mathcal{L}$; hence, in particular, each $\left(X, B_{i}\right)$ is $\mathrm{LC}$.

\section{§3. Polarized pairs}

A polarized pair is of the form $(X, B+P)$, where $(X, B)$ is a projective pair in the usual sense and $P$ is a nef $\mathbb{R}$-divisor on $X$. We will call $K_{X}+B+P$ a polarized log divisor. We say that a polarized pair $(X, B+P)$ is $L C$ (resp., $D L T$ and so forth) if $(X, B)$ is LC (resp., DLT and so forth). In this section, we will look at various questions concerning polarized pairs. Essentially, we 
would like to know how much of the birational geometry of usual pairs can be extended to polarized pairs.

Cone and contraction, flips, LMMP

For simplicity, assume that $(X, B)$ is DLT. We can run the LMMP on $K_{X}+B+P$ as follows. Let $R$ be a $\left(K_{X}+B+P\right)$-negative extremal ray. Pick an ample $\mathbb{R}$-divisor $A$ such that $\left(K_{X}+B+P+A\right) \cdot R<0$. We have $K_{X}+\Delta \sim_{\mathbb{R}} K_{X}+B+P+A$ for some boundary $\Delta$ so that $(X, \Delta)$ is DLT. Then, by the cone theorem $R$ can be contracted. This shows that the cone and contraction theorems hold for polarized pairs. Moreover, the log flip exists if $R$ defines a flipping contraction. We can continue the process; that is, if we have already obtained a model $X^{\prime}$ and if we have a $\left(K_{X^{\prime}}+B^{\prime}+P^{\prime}\right)$ negative extremal ray $R^{\prime}$, then perhaps after replacing $A$ we can make sure that $\left(K_{X^{\prime}}+B^{\prime}+P^{\prime}+A^{\prime}\right) \cdot R^{\prime}<0$ and that $\left(X, \Delta^{\prime}\right)$ is DLT, where ${ }^{\prime}$ denotes birational transform. So, $R^{\prime}$ can be contracted, and so on. The process gives an LMMP on $K_{X}+B+P$.

Question 3.1. Does the LMMP just defined terminate?

Under some mild assumptions, we will show that at least some LMMPs on $K_{X}+B+P$ terminate if every LMMP on $K_{X}+B$ terminates. In particular, we can apply this in dimension at most 3 since the latter termination is known.

TheOREM 3.2. Let $(X, B+P)$ be a $\mathbb{Q}$-factorial DLT polarized pair. Assume either that $P$ or $N=K_{X}+B-P$ is a $\mathbb{Q}$-divisor, or that $N \geq 0$ with $\operatorname{Supp} N \subseteq \operatorname{Supp} B$. Then, for any $\alpha \gg 0$, any LMMP on $K_{X}+B+\alpha P$ is P-trivial.

Proof. Here $P$-trivial means that $P$ is numerically trivial on every extremal ray in the process. First, assume that $N=K_{X}+B-P$ is a $\mathbb{Q}$-divisor. By the arguments in Section 2, we can write $P=\sum r_{i} P_{i}$, where $P_{i}$ are $\mathbb{Q}$-divisors, $r_{i}>0$ are linearly independent over $\mathbb{Q}$, and each $\left(X, B_{i}\right)$ is $\mathrm{LC}$.

Pick $\alpha>0$, and let $R$ be an extremal ray such that $\left(K_{X}+B+\alpha P\right)$. $R<0$. Then, $\left(K_{X}+B\right) \cdot R<0$ and $N \cdot R<0$. Let $\Gamma$ be an extremal curve generating $R$. (Note that $\Gamma$ is a curve generating $R$ having minimal degree with respect to some ample divisor; see [21, Definition 1] or [1, Section 3] for more details.) Then, as a consequence of the boundedness of the length of extremal rays (see [12]), we have $\left(K_{X}+B\right) \cdot \Gamma \geq-2 \operatorname{dim} X$, 
which implies that

$$
\alpha P \cdot \Gamma<-\left(K_{X}+B\right) \cdot \Gamma \leq 2 \operatorname{dim} X
$$

On the other hand, if $P_{i} \cdot \Gamma<0$, then $\left(K_{X}+B_{i}\right) \cdot \Gamma<0$, and

$$
P_{i} \cdot \Gamma=\left(K_{X}+B_{i}\right) \cdot \Gamma-N \cdot \Gamma>\left(K_{X}+B_{i}\right) \cdot \Gamma \geq-2 \operatorname{dim} X
$$

Thus, $P_{i} \cdot \Gamma \geq-2 \operatorname{dim} X$ for every $i$. Now pick a positive integer $m$ such that $m P_{i}$ is Cartier for every $i$. Then,

$$
P \cdot \Gamma=\sum r_{i} P_{i} \cdot \Gamma=\sum r_{i} \frac{n_{i}}{m},
$$

where $n_{i}$ are integers and $n_{i} \geq-2 m \operatorname{dim} X$. This implies that $P \cdot \Gamma=0$ or that $P \cdot \Gamma>\mu$, where $\mu>0$ depends only on $m$ and $r_{i}$. In particular, if $\alpha \gg 0$ (depending only on $m, r_{i}$ ), then from $\alpha P \cdot \Gamma<2 \operatorname{dim} X$ we deduce that $P \cdot \Gamma=0$.

From now on we fix $\alpha \gg 0$. By construction, $\sum r_{i} P_{i} \cdot \Gamma=P \cdot \Gamma=0$. Since the $r_{i}$ are linearly independent over $\mathbb{Q}$, we have $P_{i} \cdot \Gamma=0$ for every $i$. In particular, $\left(K_{X}+B_{i}\right) \cdot \Gamma=N \cdot \Gamma<0$ for each $i$. If $R$ defines a Mori fiber space, then the LMMP stops. Otherwise, let $X \rightarrow X^{\prime}$ be the divisorial contraction or the flip associated to $R$. Then, $K_{X^{\prime}}+B^{\prime}=P^{\prime}+N^{\prime}, P^{\prime}$ is nef, $m P_{i}$ is Cartier, and so forth, so we can apply the above arguments on $X^{\prime}$ and in this way obtain an LMMP on $K_{X}+B+\alpha P$ which is $P$-trivial in every step.

Now we treat the case when $P$ is a $\mathbb{Q}$-divisor. Pick a sufficiently large number $\alpha$. Let $m>0$ be an integer such that $m P$ is Cartier. Let $R$ be an extremal ray such that $\left(K_{X}+B+\alpha P\right) \cdot R<0$, and let $\Gamma$ be an extremal curve generating $R$. Then, $\alpha P \cdot \Gamma=\alpha(n / m)$ for some integer $n \geq 0$, and from

$$
\alpha P \cdot \Gamma<-\left(K_{X}+B\right) \cdot \Gamma \leq 2 \operatorname{dim} X
$$

we deduce that $P \cdot \Gamma=0$ since $\alpha$ is sufficiently large. The rest of the argument goes as before.

Now we come to the third case, that is, when $N \geq 0$ and $\operatorname{Supp} N \subseteq$ Supp $B$. First pick a sufficiently small $\epsilon>0$, and let $N^{\prime}$ be a $\mathbb{Q}$-divisor such that we have $(1-\epsilon) N \leq N^{\prime} \leq N$. Put $B^{\prime}=P+N^{\prime}-K_{X}$. Then, $\left(X, B^{\prime}\right)$ is DLT. By the constructions in Section 2, we can write $P=\sum r_{i} P_{i}$, where $P_{i}$ are $\mathbb{Q}$-divisors, $r_{i}>0$ are linearly independent over $\mathbb{Q},\left\|P-P_{i}\right\|$ are sufficiently small with $P-P_{i}$ supported in $\operatorname{Supp} B^{\prime}=\operatorname{Supp} B$, and $\left(X, B_{i}^{\prime}\right)$ 
are LC, where $B_{i}^{\prime}:=P_{i}+N^{\prime}-K_{X}$. Let $B_{i}:=P_{i}+N-K_{X}$. Although $\left(X, B_{i}\right)$ may not be LC, $\left(X, B_{i}-\epsilon N\right)$ is LC because $B_{i}-\epsilon N \leq B_{i}^{\prime}$. Now let $R$ be an extremal ray such that $\left(K_{X}+B+\alpha P\right) \cdot R<0$, and let $\Gamma$ be an extremal curve generating $R$. Then,

$$
P_{i} \cdot \Gamma=\left(K_{X}+B_{i}-\epsilon N\right) \cdot \Gamma-(1-\epsilon) N \cdot \Gamma \geq-2 \operatorname{dim} X
$$

The rest of the argument is similar to the case when we assumed $N$ to be a $\mathbb{Q}$-divisor.

Theorem 3.3. Let $(X, B+P)$ be a $\mathbb{Q}$-factorial DLT polarized pair. Assume either that $P$ or $N=K_{X}+B-P$ is a $\mathbb{Q}$-divisor, or that $N \geq 0$ with $\operatorname{Supp} N \subseteq \operatorname{Supp} B$. Moreover, assume that every LMMP on $K_{X}+B$ terminates. Then, for any $\alpha \geq 0$ we can run an LMMP on $K_{X}+B+\alpha P$ which terminates.

Proof. Pick a sufficiently large number $\beta>\alpha$. By Theorem 3.2, we can run an LMMP on $K_{X}+B+\beta P$ which is $P$-trivial. The LMMP is an LMMP on both $K_{X}+B+\alpha P$ and $K_{X}+B$, so it terminates by assumptions. Thus, we reach a model $Y$ on which $K_{Y}+B_{Y}+\beta P_{Y}$ is nef or there is a $\left(K_{Y}+\right.$ $\left.B_{Y}+\beta P_{Y}\right)$-negative Mori fiber space structure. In the latter case we are done, since the fiber space structure is also $\left(K_{Y}+B_{Y}+\alpha P_{Y}\right)$-negative. So, assume the former case, and by replacing $X$ with $Y$ we may assume that $K_{X}+B+\beta P$ is nef.

We will run an LMMP on $K_{X}+B$ with scaling of $\beta P$. More precisely, we replace $\beta$ with the number

$$
\min \left\{t \geq \alpha \mid K_{X}+B+t P \text { is nef }\right\}
$$

If $\beta=\alpha$, we are done. So, we can assume that $\beta>\alpha$.

Claim 3.4. There is an extremal ray $R$ such that $\left(K_{X}+B\right) \cdot R<0$ but $\left(K_{X}+B+\beta P\right) \cdot R=0$.

Proof of the Claim. For any $\left(K_{X}+B\right)$-negative extremal ray $R$ there is a positive number $t \leq \beta$ such that $\left(K_{X}+B+t P\right) \cdot R=0$. If there is such an extremal ray $R$ with $t=\beta$, then we are done. Otherwise, by definition of $\beta$, there exist a strictly increasing sequence $t_{i}$ of positive numbers approaching $\beta$, and extremal rays $R_{i}$ such that $\left(K_{X}+B\right) \cdot R_{i}<0$ but $\left(K_{X}+B+t_{i} P\right) \cdot R_{i}=0$. For each $i$, pick an extremal curve $\Gamma_{i}$ generating $R_{i}$ (see [1, Section 3]). Then, the set of the intersection numbers 
$\left\{\left(K_{X}+B\right) \cdot \Gamma_{i}\right\}_{i}$ is finite (see $[1$, Remark 3.1]). So, the intersection number

$$
P \cdot \Gamma_{i}=\frac{-\left(K_{X}+B\right) \cdot \Gamma_{i}}{t_{i}}
$$

is bounded from above and below.

If $P$ is a $\mathbb{Q}$-divisor, then there are only finitely many possibilities for the numbers $P \cdot \Gamma_{i}$. Therefore, in this case there are only finitely many possibilities for the numbers $t_{i}$, and we should have $t_{i}=\beta$ for $i \gg 0$. On the other hand, if $N$ is a $\mathbb{Q}$-divisor, then from

$$
P \cdot \Gamma_{i}=\left(K_{X}+B\right) \cdot \Gamma_{i}-N \cdot \Gamma_{i}
$$

and the boundedness of $P \cdot \Gamma_{i}$, we deduce that $N \cdot \Gamma_{i}$ is bounded; hence, there are only finitely many possibilities for the numbers $N \cdot \Gamma_{i}$. This in turn implies that there are only finitely many possibilities for the numbers $P \cdot \Gamma_{i}$; hence, $t_{i}=\beta$ for $i \gg 0$. Now assume that $N \geq 0$ with $\operatorname{Supp} N \subseteq \operatorname{Supp} B$. We will proceed similarly to the proof of [3, Lemma 3.1]. First, we replace $N$ with $(1-\epsilon) N$, and we replace $B$ with $B-\epsilon N$ for a sufficiently small $\epsilon$. Then, there is a $\mathbb{Q}$-divisor $N^{\prime}$ with the same support as $N$ and such that $\left\|N-N^{\prime}\right\|$ is sufficiently small, $\left(X, B^{\prime}\right)$ is LC where $B^{\prime}=P+N^{\prime}-K_{X}$, and $\left(K_{X}+B^{\prime}\right) \cdot \Gamma_{i}<0$ for every $i$ perhaps after replacing the sequence $t_{i}$ with an infinite subsequence. As above, the set of the intersection numbers $\left\{\left(K_{X}+B^{\prime}\right) \cdot \Gamma_{i}\right\}_{i}$ is finite. Moreover, from

$$
P \cdot \Gamma_{i}=\left(K_{X}+B^{\prime}\right) \cdot \Gamma_{i}-N^{\prime} \cdot \Gamma_{i}
$$

and the boundedness of $P \cdot \Gamma_{i}$, we deduce that $N^{\prime} \cdot \Gamma_{i}$ is also bounded; hence, there are only finitely many possibilities for $N^{\prime} \cdot \Gamma_{i}$, which in turn implies that there are only finitely many possibilities for $P \cdot \Gamma_{i}$. Hence, from $\left(K_{X}+B+t_{i} P\right) \cdot \Gamma_{i}=0$ we get $t_{i}=\beta$ for $i \gg 0$. This proves the claim.

We continue the proof of the theorem. If $R$ in the claim defines a Mori fiber space structure, we stop. Otherwise, let $X \rightarrow X^{\prime}$ be the divisorial contraction or the flip associated to $R$. Continuing the process on $X^{\prime}$ and so on produces an LMMP on $K_{X}+B$ with scaling of $\beta P$. Of course, we may lose the nefness of $P$, but we do not need it. The above LMMP is obviously an LMMP on $K_{X}+B$. So, by assumptions, it terminates. Therefore, along the way we get a model $Y$ on which $K_{Y}+B_{Y}+\alpha P_{Y}$ is nef or there is a $\left(K_{Y}+B_{Y}+\alpha P_{Y}\right)$-negative Mori fiber space structure, and we are done. 
Proof of Theorem 1.4. This follows from Theorems 3.2 and 3.3.

Proof of Corollary 1.5. Since the LMMP holds in dimension at most 3, the claim follows from Theorem 1.4.

\section{Abundance}

Although the LMMP is expected to hold for polarized pairs by the above results, abundance does not hold. Indeed, if $X$ is an elliptic curve, $B=0$, and $P$ is a nontorsion numerically trivial divisor, then $K_{X}+B+P$ is nef but not semiample. A more subtle counterexample is the following. There is a smooth projective surface $X$ which is a ruled surface over some elliptic curve with a section $S$ such that $K_{X}+2 S \sim 0$ and $S$ is nef but not semiample (see [20, Example 1.1]). Now put $B=S$, and put $P=2 S$. Then, $K_{X}+B+P \sim S$, which is nef but not semiample.

\section{Finite generation}

Similar to abundance, finite generation also fails in general, although it holds in certain interesting cases, for example, Theorem 1.6. Let $C$ be an elliptic curve, and let $Q$ be a nontorsion numerically trivial divisor. Let $X=\mathbb{P}\left(\mathcal{O}_{C} \oplus \mathcal{O}_{C}(1)\right)$, and let $g: X \rightarrow C$ be the corresponding morphism. There is a birational morphism $f: X \rightarrow Z$ which contracts a curve $S$ : the morphism is induced by the globally generated invertible sheaf $\mathcal{O}_{X}(1)$, and the morphism $S \rightarrow C$ is an isomorphism. There is also a curve $T$ disjoint from $S$ given by a general section of $\mathcal{O}_{X}(1)$ such that, again, the morphism $T \rightarrow C$ is an isomorphism. It is easy to see that $K_{X}+S+T \sim 0$. Let $B=$ $S+T$, and let $P=g^{*} Q+A$, where $A$ is the divisor corresponding to $\mathcal{O}_{X}(1)$. Then, $K_{X}+B+P$ is nef and big but not semiample; hence, its algebra is not finitely generated.

\section{Nonvanishing}

As mentioned earlier, if we take $X$ to be an elliptic curve, $B=0$, and $P$ a nontorsion numerically trivial divisor, then $K_{X}+B+P$ is nef but not semiample. Moreover, the Kodaira dimension of $K_{X}+B+P$ is $-\infty$. However, up to numerical equivalence, $K_{X}+B+P$ has nonnegative Kodaira dimension. One can then ask the following.

Question 3.5. Let $(X, B+P)$ be an LC polarized pair with $K_{X}+B+P$ pseudoeffective. Is there $M \geq 0$ such that $K_{X}+B+P \equiv M$ ?

Most probably, the answer is no. However, there are interesting cases in which the answer is yes. For example, assume that $(X, B+P)$ is a KLT 
polarized pair with $B, P$ being $\mathbb{Q}$-Cartier, and let $X \rightarrow Z$ be a morphism where $Z$ is an abelian variety and $K_{X}+B+P$ is big/ $Z$; then $K_{X}+B+P \equiv$ $M$ for some $M \geq 0$. This can be proved as in [7, Theorem 3.1] using FourierMukai transforms. (A complete proof is given in [4, Theorem 4.1].)

\section{Limiting pairs}

A limiting pair is of the form $(X, \Delta)$ where $X$ is projective and $K_{X}+\Delta$ is $\mathbb{R}$-Cartier, and there exists a sequence of boundaries $\Delta_{i}$ such that $\left(X, \Delta_{i}\right)$ are pairs in the usual sense and $K_{X}+\Delta=\lim \left(K_{X}+\Delta_{i}\right)$ in $N^{1}(X)$. We say that $(X, \Delta)$ is $L C$ if we can choose $\left(X, \Delta_{i}\right)$ to be LC.

Every LC polarized pair $(X, \Delta=B+P)$ is an LC limiting pair: we can pick ample $\mathbb{Q}$-divisors $A_{i}$ with $\lim A_{i}=0$ and choose appropriate $\Delta_{i} \sim_{\mathbb{R}}$ $B+P+A_{i}$ so that $\left(X, \Delta_{i}\right)$ are LC pairs in the usual sense and $K_{X}+\Delta=$ $\lim \left(K_{X}+\Delta_{i}\right)$.

If $(X, \Delta)$ is a limiting pair, and if $X \rightarrow Y$ is a partial LMMP on $K_{X}+\Delta$, then $\left(Y, \Delta_{Y}\right)$ is also a limiting pair. The point is that $X \rightarrow Y$ is also a partial LMMP on $K_{X}+\Delta_{i}$ for every $i \gg 0$, so the pairs $\left(Y, \Delta_{i, Y}\right)$ are all LC in the usual sense when $i \gg 0$.

The cone and contraction theorems and the existence of log flips hold for every LC limiting pair. One then wonders if a reasonable birational theory can be developed for such pairs.

\section{§4. Nakayama-Zariski decomposition with nef positive part}

Nakayama [17] defined a decomposition $D=P_{\sigma}(D)+N_{\sigma}(D)$ for any pseudoeffective $\mathbb{R}$-divisor $D$ on a smooth projective variety. We refer to this as the Nakayama-Zariski decomposition. We call $P_{\sigma}$ the positive part and $N_{\sigma}$ the negative part. We can extend it to the singular case as follows. Let $X$ be a normal projective variety, and let $D$ be a pseudoeffective $\mathbb{R}$-Cartier divisor on $X$. We define $P_{\sigma}(D)$ by taking a resolution $f: W \rightarrow X$ and letting $P_{\sigma}(D):=f_{*} P_{\sigma}\left(f^{*} D\right)$. The following lemma shows, in particular, that this is independent of the choice of the resolution.

LEMma 4.1. With $X, D, W$ as above, we have the following.

(1) $P_{\sigma}(D)$ is independent of the choice of the resolution $f$.

(2) For any $\mathbb{R}$-divisor $E \geq 0$ on $W$ which is exceptional/ $X$, we have $P_{\sigma}\left(f^{*} D+E\right)=P_{\sigma}\left(f^{*} D\right)$.

(3) If $0 \leq M$ is $\mathbb{R}$-Cartier with $\operatorname{Supp} M \subseteq \operatorname{Supp} N_{\sigma}(D)$, then $P_{\sigma}(D+M)=$ $P_{\sigma}(D)$. 
(4) If $0 \leq M \leq N_{\sigma}(D)$ is $\mathbb{R}$-Cartier, then $P_{\sigma}(D-M)=P_{\sigma}(D)$.

(5) If $\phi: X \rightarrow Y$ is a partial $D-M M P$, then $\phi_{*} P_{\sigma}(D)=P_{\sigma}\left(\phi_{*} D\right)$.

(6) If $A$ is $\mathbb{R}$-Cartier and $D+\epsilon A$ is a movable $\mathbb{R}$-divisor for each sufficiently small $\epsilon>0$, then $P_{\sigma}(D)=D$.

Proof. (1) This follows from [17, Chapter 3, Theorem 5.16].

(2) This is similar to [17, Chapter 3, Lemma 5.14]. We have

$$
P_{\sigma}\left(f^{*} D+E\right)+N_{\sigma}\left(f^{*} D+E\right)=f^{*} D+E \equiv E / X
$$

hence, $N_{\sigma}\left(f^{*} D+E\right)-E$ is anti-nef on the very general curves/ $X$ of each component of $E$, and its pushdown on $X$ is effective. Then, by Shokurov's generalized negativity lemma (see [2, Lemma 3.3]), we have $N_{\sigma}\left(f^{*} D+E\right)-$ $E \geq 0$. Therefore,

$$
P_{\sigma}\left(f^{*} D+E\right)=P_{\sigma}\left(f^{*} D\right)
$$

by [17, Chapter 3, Lemma 1.8].

(3) Let $P=P_{\sigma}(D)$, and let $N=N_{\sigma}(D)$. Choose $a>0$ so that $M \leq a N$. Then,

$$
N_{\sigma}(D+M)=N_{\sigma}(P+N+M) \leq N_{\sigma}(N+M) \leq N+M
$$

Thus, $P_{\sigma}(D+M)=P_{\sigma}(P+N+M)=P+C$ for some $0 \leq C \leq N+M$. Then,

$$
\begin{aligned}
(1+a) P & =P_{\sigma}((1+a) D)=P_{\sigma}(D+a P+a N) \\
& =P_{\sigma}(D+M+a P+a N-M) \geq P+C+a P,
\end{aligned}
$$

which is possible only if $C=0$.

(4) If $M^{\sim}$ is the birational transform of $M$ on $W$, then $M^{\sim} \leq N_{\sigma}\left(f^{*} D\right)$. By [17, Chapter 3, Lemma 1.8], $P_{\sigma}\left(f^{*} D-M^{\sim}\right)=P_{\sigma}\left(f^{*} D\right)$. Now if we let $f^{*} M=M^{\sim}+E$, then

$$
P_{\sigma}\left(f^{*} D-M^{\sim}\right)=P_{\sigma}\left(f^{*} D-f^{*} M+E\right)=P_{\sigma}\left(f^{*} D-f^{*} M\right)
$$

by $(2)$; hence, $P_{\sigma}(D)=P_{\sigma}(D-M)$.

(5) We may assume that $f: W \rightarrow X$ and $g: W \rightarrow Y$ give a common resolution. Then, $f^{*} D=g^{*} D_{Y}+E$, where $D_{Y}:=\phi_{*} D$ and $E \geq 0$ is exceptional $/ Y$. By (2) we have

$$
P_{\sigma}\left(f^{*} D\right)=P_{\sigma}\left(g^{*} D_{Y}+E\right)=P_{\sigma}\left(g^{*} D_{Y}\right)
$$


hence,

$$
\phi_{*} P_{\sigma}(D)=\phi_{*} f_{*} P_{\sigma}\left(f^{*} D\right)=\phi_{*} f_{*} P_{\sigma}\left(g^{*} D_{Y}\right)=g_{*} P_{\sigma}\left(g^{*} D_{Y}\right)=P_{\sigma}\left(D_{Y}\right) .
$$

(6) Take a strictly decreasing sequence of numbers $\epsilon_{i}>0$ with $\lim \epsilon_{i}=0$. Pick an ample divisor $G$ on $W$ so that $G+f^{*} A$ is ample. Since $D+\epsilon_{i} A$ is a movable $\mathbb{R}$-divisor, $N_{\sigma}\left(f^{*} D+\epsilon_{i}\left(f^{*} A+G\right)\right)$ is exceptional $/ X$. By going to the limit, we deduce that $N_{\sigma}\left(f^{*} D\right)$ is also exceptional $/ X$; hence, $P_{\sigma}(D)=$ $f_{*} P_{\sigma}\left(f^{*} D\right)=D$.

We will say that a pseudoeffective $\mathbb{R}$-Cartier divisor $D$ on a normal projective variety $X$ birationally has a Nakayama-Zariski decomposition with nef positive part if there is a resolution $f: W \rightarrow X$ so that $P_{\sigma}\left(f^{*} D\right)$ is nef. If $g: V \rightarrow W$ is any birational morphism from a smooth projective $V$, then $P_{\sigma}\left(g^{*} f^{*} D\right)=g^{*} P_{\sigma}\left(f^{*} D\right)$. Indeed, since $P_{\sigma}\left(f^{*} D\right)$ is nef, $P_{\sigma}\left(g^{*} f^{*} D\right) \geq$ $g^{*} P_{\sigma}\left(f^{*} D\right)$; on the other hand, in general $P_{\sigma}\left(g^{*} f^{*} D\right) \leq g^{*} P_{\sigma}\left(f^{*} D\right)$, which follows from the generalized negativity lemma (see [2, Lemma 3.3]) because $E:=g^{*} P_{\sigma}\left(f^{*} D\right)-P_{\sigma}\left(g^{*} f^{*} D\right)$ is exceptional/ $W$ and it is anti-nef on the very general curves/ $W$ of each component of $E$.

Proof of Theorem 1.1. By assumptions, there is a $\log$ resolution $f: W \rightarrow$ $X$ on which we have $f^{*}\left(K_{X}+B\right)=P+N$, where $P:=P_{\sigma}\left(f^{*}\left(K_{X}+B\right)\right)$ is nef. We can write

$$
K_{W}+B_{W}=f^{*}\left(K_{X}+B\right)+E,
$$

where $B_{W}, E \geq 0$, and $E$ is exceptional $/ X$ whose support contains each prime exceptional $/ X$ divisor $D$ on $W$ if $a(D, X, B)>0$. The pair $\left(W, B_{W}\right)$ is DLT, and a log minimal model of $\left(W, B_{W}\right)$ is also a log minimal model of $(X, B)$ by [2, Remark 2.8]. By Lemma 4.1(2),

$$
P_{\sigma}\left(K_{W}+B_{W}\right)=P_{\sigma}\left(f^{*}\left(K_{X}+B\right)+E\right)=P_{\sigma}\left(f^{*}\left(K_{X}+B\right)\right)=P .
$$

So, we can replace $(X, B)$ with $\left(W, B_{W}\right)$, and we can assume that $K_{X}+$ $B=P+N$, where $P=P_{\sigma}\left(K_{X}+B\right)$ is nef. Moreover, by taking a higher resolution, if necessary we can also assume that $(X, B+N)$ is $\log$ smooth.

First assume that $N$ is a $\mathbb{Q}$-divisor. Let $\alpha$ be a sufficiently large number. Now run an LMMP on $K_{X}+B+\alpha P$ with scaling of some ample divisor $A$. By Theorem 3.2, $P$ is numerically trivial on each extremal ray in the process; hence, the nef property of $P$ is preserved, and the LMMP is also an LMMP on $K_{X}+B$. After finitely many steps, the LMMP consists of only log flips; 
hence, we reach a model $Y$ on which $K_{Y}+B_{Y}+\alpha P_{Y}+\epsilon A_{Y}$ is a movable $\mathbb{R}$-divisor for each $\epsilon>0$.

Since

$$
N_{\sigma}\left(K_{X}+B+\alpha P+\alpha N\right)=N_{\sigma}((1+\alpha)(P+N))=(1+\alpha) N
$$

from Lemma 4.1(4) we get

$$
P_{\sigma}\left(K_{X}+B+\alpha P\right)=P_{\sigma}(P+N+\alpha P)=(1+\alpha) P .
$$

By Lemma 4.1(5),

$$
P_{\sigma}\left(K_{Y}+B_{Y}+\alpha P_{Y}\right)=\phi_{*} P_{\sigma}\left(K_{X}+B+\alpha P\right)=(1+\alpha) P_{Y},
$$

where $\phi$ is the birational map $X \rightarrow Y$. On the other hand, by Lemma 4.1(6),

$$
P_{\sigma}\left(K_{Y}+B_{Y}+\alpha P_{Y}\right)=K_{Y}+B_{Y}+\alpha P_{Y}=N_{Y}+(1+\alpha) P_{Y} .
$$

Therefore, $N_{Y}=0$ and $K_{Y}+B_{Y}=P_{Y}$, which is nef. This means that the LMMP terminates, and we get a log minimal model of $(X, B)$.

Now we treat the general case when $N$ is not necessarily a $\mathbb{Q}$-divisor. Since $(X, B+N)$ is $\log$ smooth, we can find an $\mathbb{R}$-divisor $G$ with sufficiently small coefficients such that $\operatorname{Supp} G \subseteq \operatorname{Supp} N,\left(X, B^{\prime}=B+G\right)$ is DLT, $N^{\prime}=N+G \geq 0$ is a $\mathbb{Q}$-divisor, and $\operatorname{Supp} N^{\prime}=\operatorname{Supp} N$. Note that $G$ may not be effective, but this does not cause any problem.

We get the decomposition $K_{X}+B^{\prime}=P+N^{\prime}$ where by Lemma 4.1(3)(4) we have $P=P_{\sigma}\left(K_{X}+B^{\prime}\right)$, which is nef. By the arguments above, we can run a $P$-trivial LMMP on $K_{X}+B^{\prime}$, which ends up with a log minimal model $\left(Y, B_{Y}^{\prime}\right)$. The LMMP contracts $N^{\prime}$; hence, it contracts $N$ as well. Let $f: W \rightarrow X$ and $g: W \rightarrow Y$ be a common resolution. Put $R=f^{*}\left(K_{X}+B\right)-$ $g^{*}\left(K_{Y}+B_{Y}\right)$. Since the LMMP is $P$-trivial, $f_{*} g^{*}\left(P_{Y}\right)=P$, from which we get

$$
\begin{aligned}
f_{*} R & =K_{X}+B-f_{*} g^{*}\left(K_{Y}+B_{Y}\right)=K_{X}+B-f_{*} g^{*}\left(P_{Y}\right) \\
& =K_{X}+B-P=N \geq 0 .
\end{aligned}
$$

Thus, $R \geq 0$ by the negativity lemma. Moreover, any prime divisor $D$ on $X$ contracted by $X \rightarrow Y$ is a component of $f_{*} R=N$; hence,

$$
a(D, X, B)<a\left(D, Y, B_{Y}\right) .
$$

Therefore, $\left(Y, B_{Y}\right)$ is a $\log$ minimal model of $(X, B)$. 


\section{§5. Fujita- and CKM-Zariski decompositions}

Let $D$ be an $\mathbb{R}$-Cartier divisor on a normal projective variety $X$. A FujitaZariski decomposition for $D$ is an expression $D=P+N$ such that

- $P$ is nef, $N \geq 0$, and

- if $f: W \rightarrow X$ is a birational morphism from a normal projective variety and $f^{*} D=P^{\prime}+N^{\prime}$ with $P^{\prime}$ nef and $N^{\prime} \geq 0$, then $P^{\prime} \leq f^{*} P$.

On the other hand, a $C K M-Z$ ariski decomposition for $D$ is an expression $D=P+N$ such that

- $P$ is nef, $N \geq 0$, and

- the maps $H^{0}(X,\lfloor m P\rfloor) \rightarrow H^{0}(X,\lfloor m D\rfloor)$ are isomorphisms for all $m \in \mathbb{N}$.

In both decompositions, $P$ is called the positive part.

Lemma 5.1. Let $D$ be an $\mathbb{R}$-Cartier divisor on a normal projective variety $X$ with a Fujita-Zariski decomposition $D=P+N$. Let $G$ be an $\mathbb{R}$-Cartier divisor such that $\operatorname{Supp} G \subseteq \operatorname{Supp} N$ and $N+G \geq 0$. Then, $P+N+G$ is a Fujita-Zariski decomposition of $D+G$ with $P$ the positive part.

Proof. Put $M=N+G$. Let $f: W \rightarrow X$ be a birational morphism from a normal projective variety, and assume that $f^{*}(D+G)=P^{\prime}+M^{\prime}$, where $P^{\prime}$ is nef and $M^{\prime} \geq 0$. We need to show that $f^{*} P \geq P^{\prime}$. There is $a>0$ such that $a N=M+L$ with $L \geq 0$. Then, we have

$$
\begin{aligned}
f^{*}(a D) & =a f^{*} P+a f^{*} N=a f^{*} P+f^{*} M+f^{*} L \\
& =(a-1) f^{*} P+f^{*}(D+G)+f^{*} L \\
& =(a-1) f^{*} P+P^{\prime}+M^{\prime}+f^{*} L .
\end{aligned}
$$

Since $a D=a P+a N$ is a Fujita-Zariski decomposition with $a P$ the positive part, we deduce that $a f^{*} P \geq(a-1) f^{*} P+P^{\prime}$; hence, $f^{*} P \geq P^{\prime}$.

Let $(X, B)$ be a projective KLT pair. Kawamata [11] showed that if $K_{X}+$ $B$ is a big $\mathbb{Q}$-divisor and if it has a CKM-Zariski decomposition, then the LC ring of $(X, B)$ is finitely generated; that is, $(X, B)$ has an LC model (see also Moriwaki [16] and Fujita [10]). We prove a similar result for LC pairs where we replace LC model by log minimal model.

Theorem 5.2. Let $(X, B)$ be a projective $L C$ pair such that $K_{X}+B$ is big. Then, the following are equivalent:

(1) $K_{X}+B$ birationally has a Fujita-Zariski decomposition; 
(2) $K_{X}+B$ birationally has a CKM-Zariski decomposition;

(3) $(X, B)$ has a log minimal model.

Proof. For the implication $(3) \Longrightarrow(2)$, see the proof of [3, Theorem 1.5]. For $(2) \Longrightarrow(1)$, see Prokhorov [18, Section 7].

It remains to show that $(1) \Longrightarrow(3)$. By assumptions, there is a log resolution $f: W \rightarrow X$ such that we have a Fujita-Zariski decomposition $f^{*}\left(K_{X}+\right.$ $B)=P+N$. We can write

$$
K_{W}+B_{W}=f^{*}\left(K_{X}+B\right)+E,
$$

where $B_{W}, E \geq 0$, and $E$ is exceptional $/ X$ whose support contains each prime exceptional $/ X$ divisor $D$ on $W$ if $a(D, X, B)>0$. The pair $\left(W, B_{W}\right)$ is DLT, and a log minimal model of $\left(W, B_{W}\right)$ is also a log minimal model of $(X, B)$ by $\left[2\right.$, Remark 2.8]. Moreover, $K_{W}+B_{W}=P+N+E$ is a FujitaZariski decomposition with $P$ being the positive part; indeed, assume that $g: V \rightarrow W$ is a birational morphism from a normal projective variety and that $g^{*}\left(K_{W}+B_{W}\right)=P^{\prime}+N^{\prime}$, where $P^{\prime}$ is nef and $N^{\prime} \geq 0$. Then, $g^{*} f^{*}\left(K_{X}+\right.$ $B)+g^{*} E=P^{\prime}+N^{\prime}$; hence, $N^{\prime}-g^{*} E$ is anti-nef $/ X$, and the negativity lemma shows that $N^{\prime}-g^{*} E \geq 0$. So, $g^{*} P+g^{*} N=P^{\prime}+N^{\prime}-g^{*} E$, and from this we obtain $g^{*} P \geq P^{\prime}$, which proves the claim.

By replacing $(X, B)$ with $\left(W, B_{W}\right)$, we could assume that $K_{X}+B=$ $P+N$ is a Fujita-Zariski decomposition and that $(X, B+N)$ is $\log$ smooth. Since $K_{X}+B$ is big, we can write $K_{X}+B \sim_{\mathbb{R}} A+L$, where $A$ is an ample $\mathbb{R}$-divisor and $L \geq 0$.

Assume that $N$ is a $\mathbb{Q}$-divisor. Then, by Theorem 3.2, we can run a $P$-trivial LMMP on $K_{X}+B+\alpha P$ with scaling of some multiple of $A$, for some sufficiently large number $\alpha$. We arrive at a model $Y$ on which $K_{Y}+B_{Y}+\alpha P_{Y}+\epsilon A_{Y}$ is semiample for some sufficiently small $\epsilon>0$. Then, we have

$$
\begin{aligned}
(1+\alpha+\epsilon)\left(P_{Y}+N_{Y}\right) & =(1+\alpha+\epsilon)\left(K_{Y}+B_{Y}\right) \\
& \sim_{\mathbb{R}} K_{Y}+B_{Y}+\alpha P_{Y}+\epsilon A_{Y}+\alpha N_{Y}+\epsilon L_{Y}
\end{aligned}
$$

Then, for some numerically trivial $\mathbb{R}$-divisor $Q$ we have

$$
(1+\alpha+\epsilon)\left(P_{Y}+N_{Y}\right)=Q+K_{Y}+B_{Y}+\alpha P_{Y}+\epsilon A_{Y}+\alpha N_{Y}+\epsilon L_{Y}
$$

Since $X \rightarrow Y$ was obtained as a $P$-trivial LMMP $/ Z, K_{Y}+B_{Y}=P_{Y}+N_{Y}$ is still a Fujita-Zariski decomposition by reasoning as in the second paragraph 
of this proof. So, we get

$$
(1+\alpha+\epsilon) P_{Y} \geq Q+K_{Y}+B_{Y}+\alpha P_{Y}+\epsilon A_{Y}
$$

hence,

$$
(1+\alpha+\epsilon) N_{Y} \leq \alpha N_{Y}+\epsilon L_{Y}
$$

By our choice of $\epsilon$, the latter inequality is possible only if $N_{Y}=0$, in which case $K_{Y}+B_{Y}=P_{Y}$ is nef, and we get the desired log minimal model.

Now we deal with the case when $N$ may not be a $\mathbb{Q}$-divisor. We argue as in the proof of Theorem 1.1. Pick an $\mathbb{R}$-divisor $G$ with sufficiently small coefficients such that $\operatorname{Supp} G \subseteq \operatorname{Supp} N,\left(X, B^{\prime}=B+G\right)$ is DLT, $N^{\prime}=$ $N+G \geq 0$ is a $\mathbb{Q}$-divisor, and $\operatorname{Supp} N^{\prime}=\operatorname{Supp} N$. We get the decomposition $K_{X}+B^{\prime}=P+N^{\prime}$, which is a Fujita-Zariski decomposition by Lemma 5.1, with $P$ being the positive part. By the arguments above, we can run a $P$ trivial LMMP on $K_{X}+B^{\prime}$ which ends up with a log minimal model $\left(Y, B_{Y}^{\prime}\right)$. The LMMP contracts $N^{\prime}$; hence, it contracts $N$ as well. Let $f: W \rightarrow X$ and $g: W \rightarrow Y$ be a common resolution. Put $R=f^{*}\left(K_{X}+B\right)-g^{*}\left(K_{Y}+B_{Y}\right)$. Since the LMMP is $P$-trivial, $f_{*} g^{*}\left(P_{Y}\right)=P$, from which we get

$$
\begin{aligned}
f_{*} R & =K_{X}+B-f_{*} g^{*}\left(K_{Y}+B_{Y}\right)=K_{X}+B-f_{*} g^{*}\left(P_{Y}\right) \\
& =K_{X}+B-P=N \geq 0 .
\end{aligned}
$$

Thus, $R \geq 0$ by the negativity lemma. Moreover, any prime divisor $D$ on $X$ contracted by $X \rightarrow Y$ is a component of $f_{*} R=N$; hence,

$$
a(D, X, B)<a\left(D, Y, B_{Y}\right) .
$$

Therefore, $\left(Y, B_{Y}\right)$ is a $\log$ minimal model of $(X, B)$.

We can generalize the theorem as in the following result. Recall that an $\mathbb{R}$-Cartier divisor $D$ is said to be abundant if $\kappa(D)=\kappa_{\sigma}(D)$ (see [15]), where $\kappa_{\sigma}(D)$ is the numerical Kodaira dimension defined by Nakayama [17, Chapter 5, Definition 2.5]. In particular, any big $\mathbb{R}$-Cartier divisor is abundant.

Theorem 5.3. Let $(X, B)$ be a projective $L C$ pair such that $K_{X}+B$ birationally has a Fujita-Zariski decomposition. Assume that $K_{X}+B$ is abundant. Then $(X, B)$ has a log minimal model.

Proof. We will show that the Fujita-Zariski decomposition coincides with the Nakayama-Zariski decomposition, so we can apply Theorem 1.1. By tak- 
ing a log resolution, we may assume that $(X, B)$ is DLT and that $K_{X}+B=$ $P+N$ is the Fujita-Zariski decomposition. In particular, this is also a CKMZariski decomposition; hence, $F \geq N$, where $F$ is the asymptotic fixed part $F:=\lim (1 / m)$ Fix $\left|\left\lfloor m\left(K_{X}+B\right)\right\rfloor\right|$. On the other hand, since $K_{X}+B$ is abundant, $F=N_{\sigma}\left(K_{X}+B\right)$ by [15, Proposition 6.4]. We immediately obtain the conclusion from $F \geq N \geq N_{\sigma}\left(K_{X}+B\right)=F$.

Using Theorem 1.2, we can take care of Theorem 1.6.

Proof of Theorem 1.6. If $(X, B)$ is $\mathbb{Q}$-factorial DLT, the proof is actually trivial since we can easily get rid of $P$ and the LC centers; hence, the claim reduces to the well-known finite generation for KLT pairs. But presence of LC singularities causes difficulties. Let $D:=K_{X}+B+P$, and let $A \geq 0$ be an ample $\mathbb{Q}$-divisor. Perhaps after replacing $A$ with some small multiple, by assumptions, we can write $D \sim_{\mathbb{Q}} A+L$, where $L \geq 0$ and $\operatorname{Supp}(A+L)$ does not contain any LC center of $(X, B)$. Thus, we can find a boundary $\Delta$ such that

$$
K_{X}+\Delta \sim_{\mathbb{Q}} K_{X}+B+P+\epsilon A+\epsilon L \sim_{\mathbb{Q}}(1+\epsilon) D,
$$

where $(X, \Delta)$ is LC and $\epsilon$ is a small rational number. Moreover, we may assume that the LC centers of $(X, \Delta)$ are exactly the LC centers of $(X, B)$. By replacing $(X, B+P)$ with $(X, \Delta)$, from now on we can assume that $P=0$.

Let $\left(Y, B_{Y}\right)$ be a $\mathbb{Q}$-factorial DLT blowup of $(X, B)$, and let $h: Y \rightarrow X$ be the corresponding morphism. Since $K_{X}+B$ is big and it birationally has a CKM-Zariski decomposition, $(X, B)$ has a log minimal model by Theorem 1.2. The log minimal model is a weak LC model of $\left(Y, B_{Y}\right)$. Thus, $\left(Y, B_{Y}\right)$ also has a log minimal model by [2, Corollary 3.7]. So, we can run an LMMP on $K_{Y}+B_{Y}$ with scaling of some ample divisor which ends up with a $\log$ minimal model $\left(Y^{\prime}, B_{Y^{\prime}}\right)$. By [8, Theorem 4.2], it is enough to show that $\left(Y^{\prime}, B_{Y^{\prime}}\right)$ is $\log$ abundant, that is, that the restriction of $K_{Y^{\prime}}+B_{Y^{\prime}}$ to any $\mathrm{LC}$ center of $\left(Y^{\prime}, B_{Y^{\prime}}\right)$ is abundant.

Let $S^{\prime}$ be an LC center of $\left(Y^{\prime}, B_{Y^{\prime}}\right)$. There is an LC center $S$ of $\left(Y, B_{Y}\right)$ such that $S^{\prime}$ is just the birational transform of $S$. Let $f: W \rightarrow Y$ and $g: W \rightarrow Y^{\prime}$ be a common resolution such that there is a prime divisor $T$ on $W$ mapping surjectively onto both $S$ and $S^{\prime}$. As mentioned above, we can write $K_{X}+B \sim_{\mathbb{Q}} A+L$, where $A \geq 0$ is ample, $L \geq 0$, and $\operatorname{Supp}(A+L)$ does not contain any LC center of $(X, B)$. In particular, assuming that $V$ is the image of $S$ on $X, V$ is not contained in $\operatorname{Supp}(A+L)$. Let $F$ 
be a general fiber of $S \rightarrow V$. Since $Y \rightarrow-Y^{\prime}$ is obtained by an LMMP on $K_{Y}+B_{Y} \sim_{\mathbb{Q}} h^{*}(A+L)$, and since $F$ does not intersect the support of $h^{*}(A+L), Y \rightarrow Y^{\prime}$ is an isomorphism near $F$. Therefore, if $G$ is a general fiber of $T \rightarrow V$, we have $\left.g^{*}\left(K_{Y^{\prime}}+B_{Y^{\prime}}\right)\right|_{G} \sim_{\mathbb{Q}} 0$, which implies that

$$
\kappa\left(\left.\left(K_{Y^{\prime}}+B_{Y^{\prime}}\right)\right|_{S^{\prime}}\right)=\kappa\left(\left.g^{*}\left(K_{Y^{\prime}}+B_{Y^{\prime}}\right)\right|_{T}\right) \leq \operatorname{dim} V .
$$

Let $A_{Y}=h^{*} A$, and let $L_{Y}=h^{*} L$. Since $A_{Y}$ is nef, by the negativity lemma we have $g^{*} A_{Y^{\prime}} \geq f^{*} A_{Y}$. Therefore,

$$
\begin{aligned}
\kappa\left(\left.\left(K_{Y^{\prime}}+B_{Y^{\prime}}\right)\right|_{S^{\prime}}\right) & =\kappa\left(\left.\left(A_{Y^{\prime}}+L_{Y^{\prime}}\right)\right|_{S^{\prime}}\right)=\kappa\left(\left.g^{*}\left(A_{Y^{\prime}}+L_{Y^{\prime}}\right)\right|_{T}\right) \\
& \geq \kappa\left(\left.A_{Y}\right|_{S}\right)=\operatorname{dim} V
\end{aligned}
$$

which gives the equality

$$
\kappa\left(\left.\left(K_{Y^{\prime}}+B_{Y^{\prime}}\right)\right|_{S^{\prime}}\right)=\operatorname{dim} V
$$

Finally, note that

$$
\kappa_{\sigma}\left(\left.\left(K_{Y^{\prime}}+B_{Y^{\prime}}\right)\right|_{S^{\prime}}\right) \leq \kappa_{\sigma}\left(\left.\left(K_{Y}+B_{Y}\right)\right|_{S}\right)=\kappa\left(\left.\left(K_{Y}+B_{Y}\right)\right|_{S}\right)=\operatorname{dim} V,
$$

which in particular means that

$$
\kappa_{\sigma}\left(\left.\left(K_{Y^{\prime}}+B_{Y^{\prime}}\right)\right|_{S^{\prime}}\right)=\kappa\left(\left.\left(K_{Y^{\prime}}+B_{Y^{\prime}}\right)\right|_{S^{\prime}}\right)
$$

hence, $\left.\left(K_{Y^{\prime}}+B_{Y^{\prime}}\right)\right|_{S^{\prime}}$ is abundant. Recall that $\kappa_{\sigma}$ denotes the numerical Kodaira dimension.

\section{§6. Weak Zariski decompositions}

Recall that an $\mathbb{R}$-Cartier divisor $D$ on a normal projective variety $Y$ birationally has a weak Zariski decomposition if there is a resolution $f: W \rightarrow Y$ such that $f^{*} D=P+N$, where $P$ is nef and $N \geq 0$. Assume that, for any projective LC pair $(X, B)$ of dimension at most $d$ with $K_{X}+B$ pseudoeffective birationally, we have a weak Zariski decomposition for $K_{X}+B$. Can one construct log minimal models for such pairs? In this section, we outline a strategy to tackle this problem.

Let $(X, B)$ be a projective LC pair of dimension at most $d$ with $K_{X}+$ $B$ having a weak Zariski decomposition. By taking a log resolution, we may assume that the pair is $\log$ smooth and that $K_{X}+B=P+N$ is the decomposition where $P$ is nef and $N \geq 0$. 
Step 1. By the proof of [3, Theorem 1.5], we may assume that $\operatorname{Supp} N \subseteq$ Supp $\lfloor B\rfloor$.

Step 2. Let $\alpha$ be a sufficiently large number, and run an LMMP on $K_{X}+$ $B+\alpha P$ with scaling of some ample divisor. Then, by Theorem 3.2, $P$ is trivial on each extremal ray contracted in the process, and we get an LMMP on $K_{X}+B$. By replacing $X$, we may assume that the LMMP consists of only flips. We should show that this LMMP terminates by using special termination arguments. If $S$ is a component of $\lfloor B\rfloor$, and if we put $K_{S}+B_{S}=$ $\left.\left(K_{X}+B\right)\right|_{S}$ and $P_{S}=\left.P\right|_{S}$, then we need to show that the induced LMMP on $K_{S}+B_{S}+\alpha P_{S}$ terminates. This is obviously related to the material in Section 3. This should be somehow derived from existence of weak Zariski decompositions and log minimal models in dimension less than $d$.

Step 3. If step 2 is done successfully, then we can assume that $K_{X}+$ $B+\alpha P$ is nef. Next, run an LMMP on $K_{X}+B$ with scaling of $\alpha P$ as in the proof of Theorem 3.3. Again as in step 2, we need to use special termination arguments to show that the LMMP terminates. In other words, for a component $S$ of $\lfloor B\rfloor$ we have $K_{S}+B_{S}+\alpha P_{S}$ nef, and we need to show that the induced LMMP on $K_{S}+B_{S}$ with scaling of $\alpha P_{S}$ terminates. As mentioned in step 2, this is related to Section 3.

In order to prove the terminations required in steps 2 and 3 , we probably need to generalize [1, Theorem 1.5] to the setting of polarized pairs. There are indications that in the strategy above it might be better to start with a polarized pair rather than a usual pair $(X, B)$.

Acknowledgments. The authors would like to thank Yoshinori Gongyo for useful discussions and one of the referees for valuable comments and corrections. Birkar's work was partially supported by a Leverhulme grant. Zhengyu Hu was supported by Engineering and Physical Sciences Research Council grant EP/I004130/1.

\section{REFERENCES}

[1] C. Birkar, On existence of log minimal models, II, J. Reine Angew. Math. 658 (2011), 99-113. MR 2831514. DOI 10.1515/CRELLE.2011.062.

$[2] \longrightarrow$, Existence of log canonical flips and a special LMMP, Publ. Math. Inst. Hautes Études Sci. 115 (2012), 325-368. MR 2929730. DOI 10.1007/s10240-012-0039-5.

[3] - On existence of log minimal models and weak Zariski decompositions, Math. Ann. 354 (2012), 787-799. MR 2965261. DOI 10.1007/s00208-011-0756-y.

[4] C. Birkar and J. A. Chen, Varieties fibred over abelian varieties with fibres of log general type, preprint, arXiv:1311.7396v1 [math.AG]. 
[5] C. Birkar and Y. Chen, Images of manifolds with semi-ample anti-canonical divisor, to appear in J. Algebraic Geom., preprint, arXiv:1207.4070v1 [math.AG].

[6] S. Cacciola, On the semiampleness of the positive part of CKM Zariski decompositions, Math. Proc. Cambridge Philos. Soc. 156 (2014), 1-23. MR 3144208. DOI $10.1017 /$ S0305004113000509.

[7] F. Campana, J. A. Chen, and T. Peternell, Strictly nef divisors, Math. Ann. 342 (2008), 565-585. MR 2430991. DOI 10.1007/s00208-008-0248-x.

[8] O. Fujino and Y. Gongyo, Log pluricanonical representations and abundance conjecture, preprint, arXiv:1104.0361v3 [math.AG].

[9] O. Fujino and S. Mori, A canonical bundle formula, J. Differential Geom. 56 (2000), 167-188. MR 1863025.

[10] T. Fujita, Zariski decomposition and canonical rings of elliptic threefolds, J. Math. Soc. Japan 38 (1986), 19-37. MR 0816221. DOI 10.2969/jmsj/03810019.

[11] Y. Kawamata, "The Zariski decomposition of log-canonical divisors" in Algebraic Geometry, Bowdoin, 1985, Pt. 1 (Brunswick, Maine, 1985), Proc. Sympos. Pure Math. 46, Amer. Math. Soc., Providence, 1987, 425-433. MR 0927965.

[12] - On the length of an extremal rational curve, Invent. Math. 105 (1991), 609611. MR 1117153. DOI 10.1007/BF01232281.

[13] - Subadjunction of log canonical divisors, II, Amer. J. Math. 120 (1998), 893-899. MR 1646046.

[14] J. Kollár and S. Mori, Birational Geometry of Algebraic Varieties, Cambridge Tracts in Math. 134, Cambridge University Press, Cambridge, 1998. MR 1658959. DOI 10.1017/CBO9780511662560.

[15] B. Lehmann, On Eckl's pseudo-effective reduction map, Trans. Amer. Math. Soc. 366, no. 3 (2014), 1525-1549. MR 3145741.

[16] A. Moriwaki, Semiampleness of the numerically effective part of the Zariski decomposition, J. Math. Kyoto Univ. 26 (1986), 465-481. MR 0857230.

[17] N. Nakayama, Zariski-Decomposition and Abundance, MSJ Mem. 14, Math. Soc. Japan, Tokyo, 2004. MR 2104208.

[18] Y. G. Prokhorov, On the Zariski decomposition problem (in Russian), Tr. Mat. Inst. Steklova 240 (2003), 43-72; English translation in Proc. Steklov Inst. Math. 240 (2003), 37-65. MR 1993748.

[19] V. V. Shokurov, 3-fold log models, J. Math. Sci. (N.Y.) 81 (1996), 2667-2699. MR 1420223. DOI 10.1007/BF02362335.

[20] — Complements on surfaces, J. Math. Sci. (N.Y.) 102 (2000), 3876-3932. MR 1794169. DOI 10.1007/BF02984106.

[21] - Letters of a bi-rationalist, VII: Ordered termination (in Russian), Tr. Mat. Inst. Steklova 264 (2009), 184-208; English translation in Proc. Steklov Inst. Math. 264 (2009), 178-200. MR 2590847. DOI 10.1134/S0081543809010192.

Caucher Birkar

DPMMS, Centre for Mathematical Sciences

Cambridge University

Cambridge CB3 OWB

United Kingdom

c. birkar@dpmms.cam.ac.uk 
Zhengyu $\mathrm{Hu}$

DPMMS, Centre for Mathematical Sciences

Cambridge University

Cambridge CBз $0 \mathrm{WB}$

United Kingdom

zh262@dpmms.cam.ac.uk 